\title{
Participatory Methodology for Designing an Agroecological Transition at Local Level
}

\author{
Elise Audouin, Jacques-Eric Bergez, and Olivier Therond
}

\begin{abstract}
The purpose of the TATA-BOX project was to develop a toolbox to support local stakeholders in the design of an agroecological transition at local level. A participatory process based on existing conceptual and methodological frameworks was developed for the design of new configurations of stakeholders and resource systems in the farming systems, supply-chains and natural resources management that were to form a new agroecological territorial system. This process, presented here, was adapted and tested on two adjacent territories in south-western France. It was structured around three main stakeholders' workshops to support the holistic diagnosis, the design of a normative vision, and the backcasting approach of the transition pathway. We describe the participatory methods and the multimodal intermediary tools used to support the collective design of the agroecological transition. We also present the main turnkey outcomes of the design process for local stakeholders, including shared diagnosis, vision for an agroecological territorial system in 2025, and a projected action plan for transition from the initial to the desired agriculture and associated governance structures. Finally, we discuss the limits of the process and the conditions that would enable stakeholders to implement the transition, by reducing remaining uncertainties.
\end{abstract}

\section{Introduction}

Agroecological transition (AET), i.e. the development of an agriculture based on diversified agricultural systems and associated ecosystem services, can be seen as an innovation process towards sustainable agriculture. It involves a complex

\author{
E. Audouin $(\bowtie) \cdot$ J.-E. Bergez \\ AGIR, Université de Toulouse, INRA, Castanet-Tolosan, France \\ e-mail: elise.audouin@inra.fr; jacques-eric.bergez@inra.fr \\ O. Therond \\ LAE, Université de Lorraine, INRA, Colmar, France \\ e-mail: olivier.therond@inra.fr
}


co-evolution of technological, social, economic and institutional dimensions, and depends on appropriate management and fostering of interactions between stakeholders of the farming system, the supply chain, and natural resources management at local level (Duru et al. 2015). The latter challenge calls into question the role and organisation of research in supporting the AET. The development of action-research and transformative post-normal science is intended to address this issue. According to Cash et al. (2003), the effectiveness of scientific inputs can be evaluated against three criteria: (i) the impact of science on how issues are defined; (ii) the production of useful information for society i.e. credible, salient and legitimate information; and (iii) a strong interface between scientists and stakeholders by means of effective communication.

The complexity, management challenges and societal demands of AET led some of the scientists towards a progressive change of paradigm (Pretty 1995; Lane 1998). Emergent properties of this transformative research are: (i) a holistic approach, and (ii) changes in the researcher's position to rely more on local empirical knowledges in order to create transition dynamics adapted to and accepted by the intended actors. In parallel, civil society has called for more involvement in local policy making towards a democratic ideal, targeting more integration of local perspectives into development strategies (Pinto-Correia et al. 2006; Shucksmith 2010).

Accordingly, an increasing number of recent research projects or studies dealing with societal issues, like the AET, include participatory approaches. They generally share common concepts such as systems thinking, inter-disciplinarity, and multistakeholder representation.

Among them, the TATA-BOX project - based on Duru, Therond and Fares' (2015 - hereafter denoted as DTF, chapter "TATA-BOX at a Glance") conceptual and methodological frameworks - was intended to develop an operational participatory methodology to support stakeholders in thinking and designing an AET at local level. However, even if these authors developed conceptual and methodological frameworks tailored to deal efficiently with AET challenges at local level, they did not provide operational procedures and tools to support stakeholders in the design process. Yet these operational dimensions determine meaningful knowledge-sharing and collaboration between stakeholders. They have to be designed: (i) to foster creative pathways towards sustainability; (ii) to reach agreements and foster dynamics that facilitate transition; and (iii) to generate stakeholders' engagement towards change (Checkland and Poulter 2006).

In other words, the TATA-BOX project effected a transformation from AET design theory to operational and effective practices. Which methods and tools do actually support stakeholders? How do we evaluate action research outcomes?

As a methodological project, TATA-BOX aimed to: (i) provide researchers with new perspectives on procedures to support territorial actors in the design of AET; and (ii) provide feedback on the outcomes of the project's methodology.

The objective of this chapter is to provide an overview of how a conceptual and methodological framework for AET has been processed as an operational procedure with methods and tools, and what resulted from their implementation in real local case studies. Going back from practice to theory, implementation feedback is used 
as a basis to assess how the operational procedure could actually support the complexity and management issues inherent to AET.

\section{Material and Methods}

\section{Theoretical and Methodological Frameworks}

In the theoretical framework proposed by DTF (2015), AET is defined as a process that implies three interacting domains at local scale, each of which is characterised by specific stakeholders and material resources:

- the farming system, in which farmers manage resources like land, water, infrastructures, labour, inputs, biodiversity, and semi-natural landscape features;

- the socio-technical system consisting of supply chains in which stakeholders manage resources like stocking infrastructure, agricultural products, operating standards, and production standards;

- the socio-ecological system consisting of territorial resource management arenas in which a diversity of stakeholders, including farmers, manage natural resources such as soil, water, labour, biodiversity, natural and semi-natural landscape features, artificial infrastructures, legislation and operating standards (cf. Fig. 1)



Fig. 1 Duru, Therond, Fares' conceptual framework (DTF 2015)

Local agriculture as a system of stakeholders managing three types of material resource systems through information technologies. The system of stakeholders consists of farmers and other stakeholders involved in supply chains and management of natural resources, with cognitive resources (e.g. beliefs, values, individual strategies) and whose behaviour is determined by informal norms and agreements (another type of cognitive resource) and formal rules. The tetrahedron reflects local agricultural development's reliance on interactions between its four dimensions. Each edge of the tetrahedron (double arrows) corresponds to a diversity of information technologies used to manage material resources and concrete management processes within a variety of farming systems, supply chains and natural resource management institutions 
This conceptual framework highlights the critical role of the stakeholder system emerging from interactions between and the multiple roles of actors managing these three domains. The stakeholders of these three sub-systems develop specific knowledge and management strategies.

Considering this conceptual framework, DTF (2015) proposed a 5-step participatory design process to support local stakeholders in this transition design process (cf. chapters "General Introduction" and "TATA-BOX at a Glance") (Fig. 2):

(i) analyse the current situation: this step sets the transition arena, defines the issue, identifies key stakeholders and causality chains (Duru et al. 2015). For

Social and biological diversities and connectivities Ecosystem services

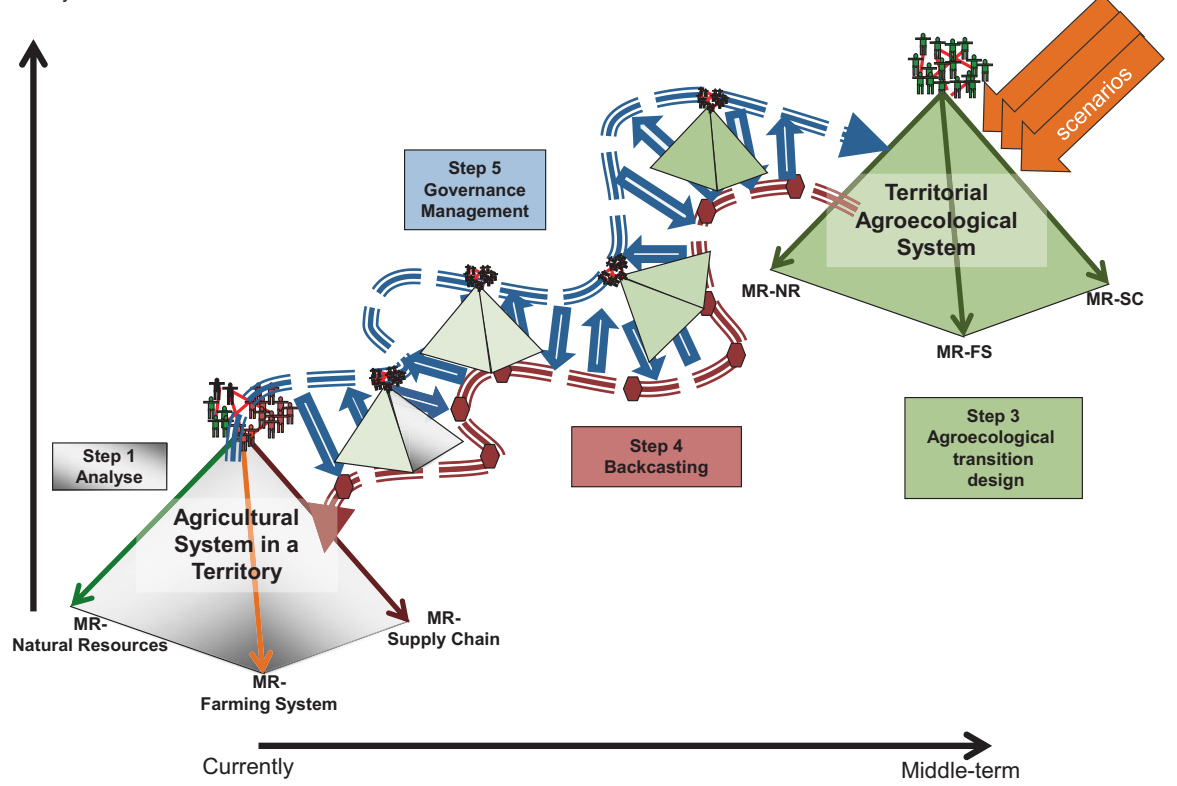

Fig. 2 DTF Methodological framework (Duru et al. 2015)

Participatory design methodology of "territorial biodiversity-based agriculture" and the transition from the current situation to this new form of agriculture. This methodology is driven by Participatory-Design Facilitator-Scientists who manage and steer a multi-stakeholder group ("transition arena") that includes stakeholders from the three management domains (farming systems, supply chains and natural resources) with key knowledge about the functioning of local agriculture. This participatory methodology is composed of five steps: (1) co-analysis of the current situation: the system of stakeholders and their material resources (MR); (2) co-identification of future changes exogenous to local agriculture, which can determine its future; (3) co-design of the expected territorial biodiversity-based agriculture; (4) co-design of the transition (pathway) from the current situation to territorial biodiversity-based agriculture (the reverse arrow indicates a backcasting approach); and (5) co-design of governance structures and adaptive management strategies enabling stakeholders to guide the transition they designed. Each step must be performed by considering and integrating interactions between farming systems, supply chains and natural resource management 
this purpose, it identifies stakeholders, resources, human-driven actions, and ecological processes that have a decisive influence on the functioning of farms, supply chains, and natural-resource management.

(ii) develop scenarios of major exogenous forces (explorative forecasting): this step identifies external changes that could affect the local territory. The identification of these changes can be based on a morphological approach that addresses the potential constraints the territory could suffer from and the potential opportunities that could impact it positively.

(iii) design a desired Territorial AgroEcological System - TAES (normative foresight): this step designs a new organisation of the local agriculture that meets local stakeholders' expectations, considering current local issues and scenarios of exogenous forces. For this purpose, graphical tools (conceptual diagrams, pictures, cognitive maps) are used iteratively. Iteration fosters innovation and progressively improves the design of scenarios with cycles of propositions and prediction of potential impacts of the proposed innovations.

(iv) design the transition pathway between the TAES and the current situation (backcasting): this step identifies the most important conditions for progressing step-by-step in the transition pathway, as well as the decisive changes and their impact on the whole system. Settings of monitoring criteria are also determined.

(v) identify the governance structure and management strategy needed to steer the transition: this step identifies governance structures and adaptive management strategies to steer and manage the transition pathway. It starts with the hypothesis that multi-stakeholders and polycentric subsystems of governance with a variety of coordination modes would be adapted to deal with the particularities of the AET (Biggs et al. 2012; Duru et al. 2015) (cf. chapter "Towards an Integrated Framework for the Governance of a Territorialised Agroecological Transition").

\section{Partnership Between Researchers and Local Authorities}

\section{Project Team and Organisation}

Forty-two researchers formed the project team. They came from various disciplines (agronomy, computer science, informatics, economy, ergonomics, management science, and sociology); from six French research organisations (CNAM, ENSAT, ENSFEA, INRA, IRSTEA, UTT ${ }^{1}$ ).

\footnotetext{
${ }^{1}$ CNAM $=$ National Conservatory of Arts and Crafts; ENSAT $=$ National High School for Agronomy of Toulouse; ENSFEA = National High School of Agricultural Training and Education; INRA = National Institute for Agricultural Research; IRSTEA = National Research Institute of Science and Technology for Environment and Agriculture; UTT = University of Technology of Troyes.
} 
The researchers were divided up into six working groups. The first three (knowledge dynamics, integrated assessment, crop-livestock farming) were set the task of providing reflective and scientific bases for AET. The fourth group was in charge of the operationalisation of the conceptual framework into process, methods and tools, along with their application on territories. This group benefited from the inputs from theoretical and empirical outcomes (from case-studies) of the first three groups, and proposals from the fifth group working on information and communication technologies (ICT) (cf. chapter "Information and Communication Technology (ICT) and the Agroecological Transition"). The last group was in charge of a reflexive analysis on the organisation of the research project ("How does the research team produce its outputs"?, cf. chapter "Towards a Reflective Approach to Research Project Management") and of the participatory methodology ("How relevant are the process, the methods, tools and the resulting products? What are the impacts on the territories?", cf. chapter "Evaluation of the Operationalisation of the TATA-BOX Process"). Accordingly, the work of the fourth group was a continuous interdisciplinary development process based on outcomes of the five other groups of the TATA-BOX project.

\section{Case Studies and Time Scale}

French government agencies for regional and rural development, named PETR, ${ }^{2}$ were chosen as a relevant scale to implement a design process of transition towards a territorial agroecological system (tTAES). This is a public institution scale used in territorial development planning for inter-municipalities. PETR local authorities are in charge of economic, ecological and cultural development, land use planning $\left(\mathrm{SCoT}^{3}\right)$, and ecological transition. They act at an intermediate scale: larger than the municipality but smaller than the French département (FADN NUTS III, http:// ec.europa.eu/agriculture/rica/). Inhabitants of the PETR share a common identity but they manage heterogeneous resources requiring varying degrees of cooperation between one another.

The PETR of Centre Ouest Aveyron (129 municipalities, $2998 \mathrm{~km}^{2}$ ) and the PETR of Midi-Quercy (48 municipalities, $1192 \mathrm{~km}^{2}$ ) were selected because: (i) these adjacent territories share a common key water resource: the Aveyron River (upstream and downstream respectively); and (ii) they could use territories' complementarities as a catalyst for transition, e.g. to organise interactions between cropand livestock-oriented farming systems (cf. details in Moraine et al. 2017). Their topographical, geological and landscape features are contrasted. Farming types evolve according to a gradient, from prevailing grassland-based upstream to rainfed crop-livestock systems (middle stream), to prevailing irrigated cropping and orchards downstream. Emblematic agricultural products are sheep cheese in Centre Ouest Aveyron and apple in Midi-Quercy (cf. Fig. 3).

\footnotetext{
${ }^{2}$ Territorial and Rural Balance Pole.

${ }^{3}$ Territorial Coherence Scheme.
} 


\section{Municipal farming types}



Fig. 3 Prevailing farm types map at municipal scale for Midi-Pyrénées region, AGRESTE 2010. The framed area indicates the position of the two selected PETR

Medium-term horizon scale of 10-15 years was proposed to local stakeholders to balance longterm agroecological transition issues with classical short-term issues managed by local stakeholders in their current projects

These two PETR signified their keen interest in developing a participatory process to design an AET at local level and, accordingly, became local partners for the TATA-BOX project.

\section{Stakeholder Analysis and Involvement}

In both territories, representative stakeholders were targeted to participate in the workshops. The operational goal was to constitute a "transition arena" along the process, i.e. a relatively small group of innovation-oriented stakeholders who reached consensus about the need and opportunity for systemic changes, and engaged in a process of social learning about future possibilities and opportunities. These specific stakeholders: (i) questioned the limits of efficiency-/ 
substitution-based agriculture; (ii) wanted to forecast which activities required changes to promote biodiversity-based agriculture; or (iii) wanted to take part in already implemented biodiversity-based agricultural systems and associated innovations (Duru et al. 2015; Foxon et al. 2009).

Representative stakeholders' identification relied on a classical stakeholder analysis (Grimble and Wellard 1997) based on key stakeholders' network mapping. The stakeholders' network was identified on the basis of the researchers' exploratory interviews with the local key actors, and the scientific knowledge drawn from their previous work in these fields. We used an interest/impact diagram to classify local stakeholders according to their willingness and their potential impact on AET (Therond et al. 2010). A third dimension taken into account in our analysis was stakeholders' position regarding AET (pro or con) (cf. Fig. 4).

This diagram was used as a decision support tool with local PETR partners to build a stakeholders database for the participatory process. In order to ensure the representativeness of the participatory process outcomes and thus to ensure its relevance, all diagram stakeholder categories were contacted: favourable, unfavour-



Fig. 4 Interest/Impact analysis diagram for Tarn-Aveyron watershed Stakeholders related to local agroecological transition are listed. They are positioned according to their interest (ordinate) in or their impact (abscissa) on Territorial Agroecological Transition. Finally, their position on participatory processes of Territorial Agroecological Transition is assessed and formalised with colours: pro (green), neutral (black), against (red) 
able and neutral stakeholders, with low, medium or high interest or impact. We finally gathered 57 heterogeneous workshop participants in total for both territories:

- Farmers (organic, conservation agriculture, conventional)

- Farmer groups (conventional and organic)

- Civil society (consumers or environmental associations)

- Farming advisory groups (conventional and organic)

- Territorial planning institutions

- Supply chain and retailers: conventional large chains (cooperative, suppliers), certification labels, short chains

\section{Scientific Design of the Participatory Methodology}

The participatory methodology was co-designed by the fourth group of the TATABOX project in 20 work sessions, from January 2014 to May 2017. We collectively discussed how to operationalise a multi-level and multi-domain participatory approach along a sequence of participatory workshops. For each workshop, the team set: specific explicit goals, a customised programme, and associated methods and tools for each step of the programme. Considering the methodological framework of DTF (2015), the operational participatory methodology was built step by step, in relation to the results, outcomes and feedback on the organisation, methods and tools of each workshop (7 workshops in total) with the local stakeholders.

The scientific process of the methodology design benefited from regular inputs from territorial experts on local issues for AET: 7 interface times with 21 local stakeholders in total. For example, the TATA-BOX process started with an immersion of researchers in the studied territories. The journey included transects, meetings with key stakeholders of agroecological initiatives to collect their testimonies and determine local needs, with a view to expanding the transition movement. Interface times were systematically organised between workshops to analyse results and new needs, and to adapt the next workshop. The outcomes of each workshop were formalised into three reports available for both workshop participants and other local stakeholders. The Scientist-Territory interface was completed by a biannual newsletter containing regular updates on project outputs and events.

For the last workshop, we intensified local partners' involvement in workshop organisation and facilitation in order to progressively transfer to them the responsibility of transition design and management process (cf. Fig. 5). 


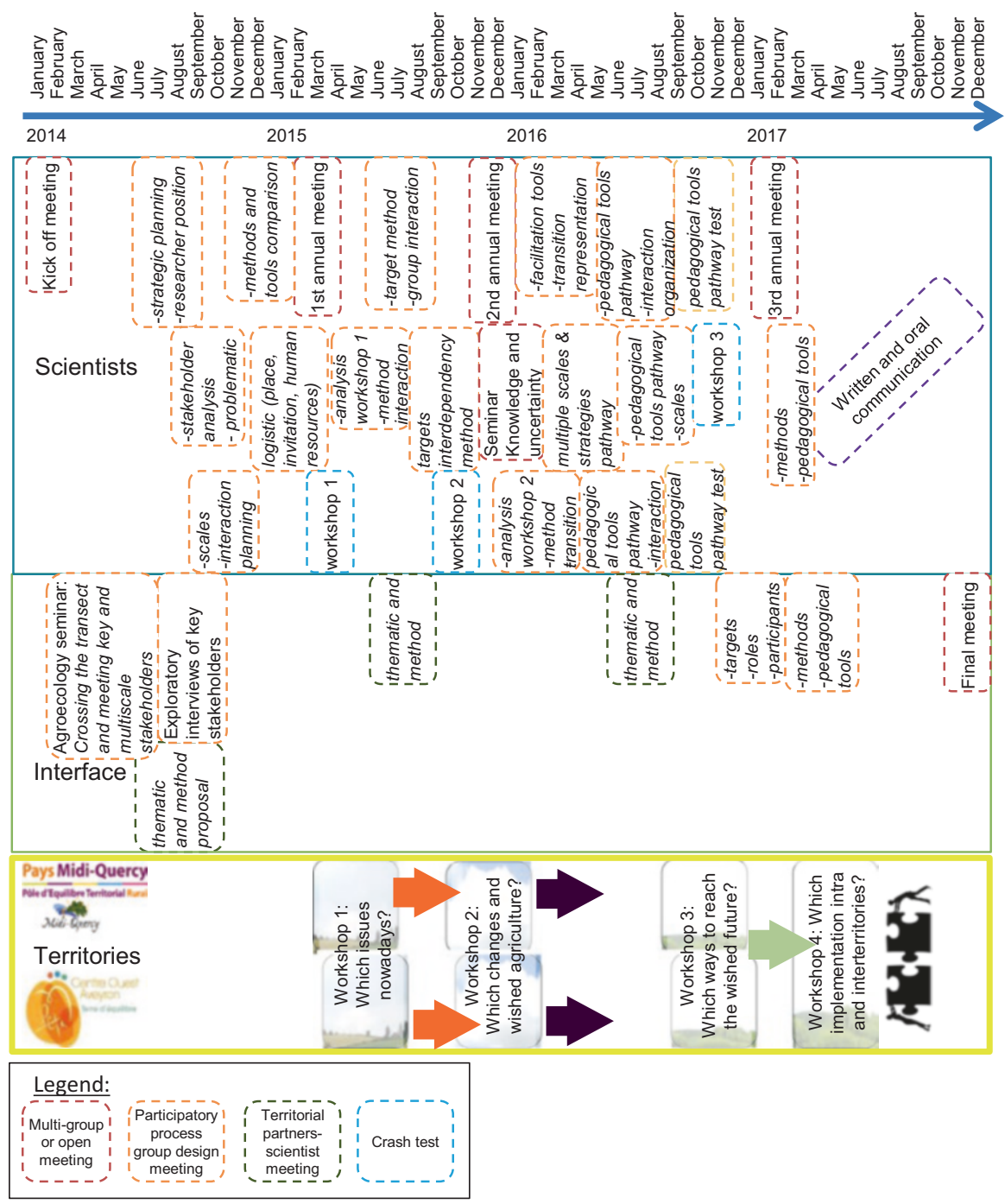

Fig. 5 Main collective reflection sessions, divided between the scientists' sphere, the territorial sphere, and the interface between them

\section{Participatory Guidelines}

\section{Participatory Action Research Guidelines}

For all workshops, we adopted 5 basic principles (Bryson et al. 2012; Vergne 2013; Jordan and Kapoor 2016): 
- transparency on targets and frame: the project aims at developing and testing supporting methods for AET design. The responsibility for the implementation of the outcomes must belong to local stakeholders; in other words, it is up to the local stakeholders to implement and manage the designed transition;

- equity: the process, methods and tools are open to the integration of local stakeholders' values and proposals;

- inclusion: the process is based on methods favouring local stakeholder involvement;

- relevance: the process must provide an added-value to the current situation; the outcomes must result in operational impacts and local stakeholder empowerment;

- neutrality: researchers propose methods and tools to facilitate stakeholders' interactions; they neither propose actions for the territory considered, nor define what exactly "agroecology" means.

\section{Participatory Methods and Tools}

Throughout the design process we used and developed methods and tools to foster mutual understanding of stakeholders' representations, knowledge exchange and creative thinking and innovation.

Techniques like icebreakers were used to develop an informal work atmosphere and thus to foster creativity and cooperation. Other techniques were used to balance speaking time and proposals among the different workshop participants. For example, in a card-sorting exercise, participants were invited to write their proposals, one per card, and then to present them one participant at a time during iterative roundtables.

We developed a dedicated communication medium, i.e. intermediary/boundary objects to open representation frames and boost creativity (e.g. drawing, maps and card games; Vinck 2009, 2011). When cumulated, the diversity of communication mediums enabled various participants with different behaviours and logics to understand and therefore to contribute to the exercises. Some participants will be more confident with oral communication, while others prefer written communication and will require visual representations to express their ideas. We most often proposed a combination of representation modes to ensure that each stakeholder would find a way to participate.

In addition, some tools were developed to enable participants to materialise the detailed description of their proposals by representing all information categories that should be informed (cf. section "Intermediary tools").

Finally, in order to conserve a maximum amount of information from one step to the next, intermediary tools were developed to facilitate the use of one workshop's outcomes in the next workshop. Here, each intermediary tool corresponds to a representation at different scales of empirical and collective knowledge (Audouin et al. 2018a). 


\section{Results}

\section{Methodological Results}

\section{Participatory Process}

\section{Process}

The DTF methodological framework includes an iterative cycle of 5 steps (cf. section "Theoretical and methodological frameworks"). The authors highlighted the fact that this segmentation is theoretical since the steps are interconnected and interdependent. While translating the original methodological frameworks into operational procedures, in a 4-year participatory process, researchers had to deal with several constraints and objectives:

- devote the first year to the analysis of the territorial context, the development of partnerships, and strategic planning: refining the process targets, identifying key stakeholders, etc.;

- limit the duration of the participatory process to facilitate continuous involvement of stakeholders throughout the process;

- avoid excessively frequent meetings with stakeholders, some of whom have limited availability;

- respect a minimum duration between workshops to allow time for their analysis and the step-by-step design of their methodology.

Due to these different constraints we opted for a total of three workshops on an 18-month basis. This process corresponded to a single iteration of the DTF methodological framework cycle (cf. section "Theoretical and methodological frameworks"). Each workshop was 1 day long. The specific targets of the workshops were to: (1) develop a shared analysis of the current situation and issues of local agriculture; (2) co-design a desired TAES resilient to future exogenous changes, involving the collective development of shared goals and visions; and (3) co-design the transition pathway to reach the future vision, with special attention paid to governance. Finally, at the workshop participants' request, we organised a last workshop to support territorial partners in managing the outcomes of design process and transition plan implementation. This step also resulted in the identification of territories' complementarities and potential synergies, in order to facilitate the implementation of action plans.

\section{Methods to Foster Participants' Interaction}

We organised participants' interactions at two different scales: during the process, during workshops, and during work sequences in workshops. In line with the objective of each work sequence, we grouped participants together either in diversified 
groups, ensuring the presence of representatives of each domain (farming system, supply chain, natural resources management, cf. section "Theoretical and methodological frameworks") or, in contrast, specialised in a domain. During the workshop and throughout the whole design process we organised interactions through four key sequences: (i) synchronisation of knowledge in plenary sessions; (ii) exploration of the space of possible options (divergence) and selection of the most interesting ones, in diversified groups; (iii) in-depth description of options by domain group (deepening); and (iv) consistency analysis of described options across domains (convergence). Divergence sessions corresponded to free expression and comparing of multiple points of view. Further reflection was held in mono-domain groups, to take advantage of participants' expert assessments in each domain. Convergence sessions corresponded to agreements and stabilisation of ideas by comparing domain insights in trans-domain groups (cf. Fig. 6).

Within a workshop, articulation between plenary sessions and group sessions, and more specifically between mono-domain and trans-domain group sessions, encouraged participants to foster a multi-domain and multi-level approach to the investigated AET. The articulation between these group session layouts allowed them to go back and forth in scale and level during each workshop.



Fig. 6 Participants' interaction throughout the participatory process. The process of Divergence Deepening - Convergence was used during the three workshops. However, depending on the goal of the workshop, this process was managed on separated or mixed domains (FS/SC/NR) to emphasise a tendency towards either Divergence, or Deepening, or Convergence 


\section{Tools for Mutual Understanding, Innovation and Cooperation}

The main outputs of the fourth group of the project, dedicated to step-by-step design, are represented in the table below. Table 1 details process planning in terms of a global dynamic, the step-by-step organisation of the participants' interaction, the facilitation methods, and the intermediary tools.

When designing a workshop, in addition to organisation layout, attention was paid to creating a gradual evolution from multiple general ideas in the divergence session, to empirical knowledge during the deepening and convergence sessions.

The first workshop was dedicated to the development of a shared representation of local agricultural issues. It alternated plenary sessions with trans-domain group session. The first plenary session included researchers' presentation of local issues from previous studies. The group session was then dedicated to the formalisation of problems to solve, assets to maintain, and opportunities to take. The second plenary session was organised to share and broaden the groups' outputs. A description of the issues was then refined by a trans-domain perspective and spatial representation exercise. A plenary session shared and gathered the groups' outputs (cf. Table 1). In this workshop a shared representation of current agriculture issues was progressively developed.

The second workshop was devoted to the collective description of targets for 2025 's local agriculture. It alternated plenary sessions with group sessions. The first plenary session synchronised participants' knowledge about local agricultural issues defined during Workshop 1, and the main exogenous forces that could influence the future of local agriculture. These forces were presented by the researchers and then discussed with the participants. To develop consensus about territorial targets, the first session was devoted to the identification of general targets in transdomain groups. The mono-domain groups then broke down these general targets into specific targets, i.e. targets concerning the specific issues of these domains (cf. Table 1). In a plenary session the groups' outputs were pooled within a common share vision for local agriculture in 2025 (cf. Table 1).

The third workshop was devoted to the description of transition pathways, i.e. to the co-design of pathways from the current state (Workshop 1) to the desired future state (Workshop 2) and to the identification of the subsequent forms of governance. The workshop started by a plenary session to synchronise participants' knowledge on the desired future state developed during Workshop 2. A trans-domain session, to take advantage of participants' complementarity, then sequenced targets to develop general action plan strategies. The participants posted the pathways of each target with milestones such as intermediary states, monitoring indicators, and modes of governance (cf. Table 1). A plenary session shared and gathered the groups' outputs.

This process progressively deepens considered scales and domain specificities, evolving from "general targets" to "domain-specific targets" in Workshop 2, to target-detailed pathways in Workshop 3.

This pattern was also observed in the types of representation. Divergence sessions were based on abstract representations to foster creativity: brainstorming or 


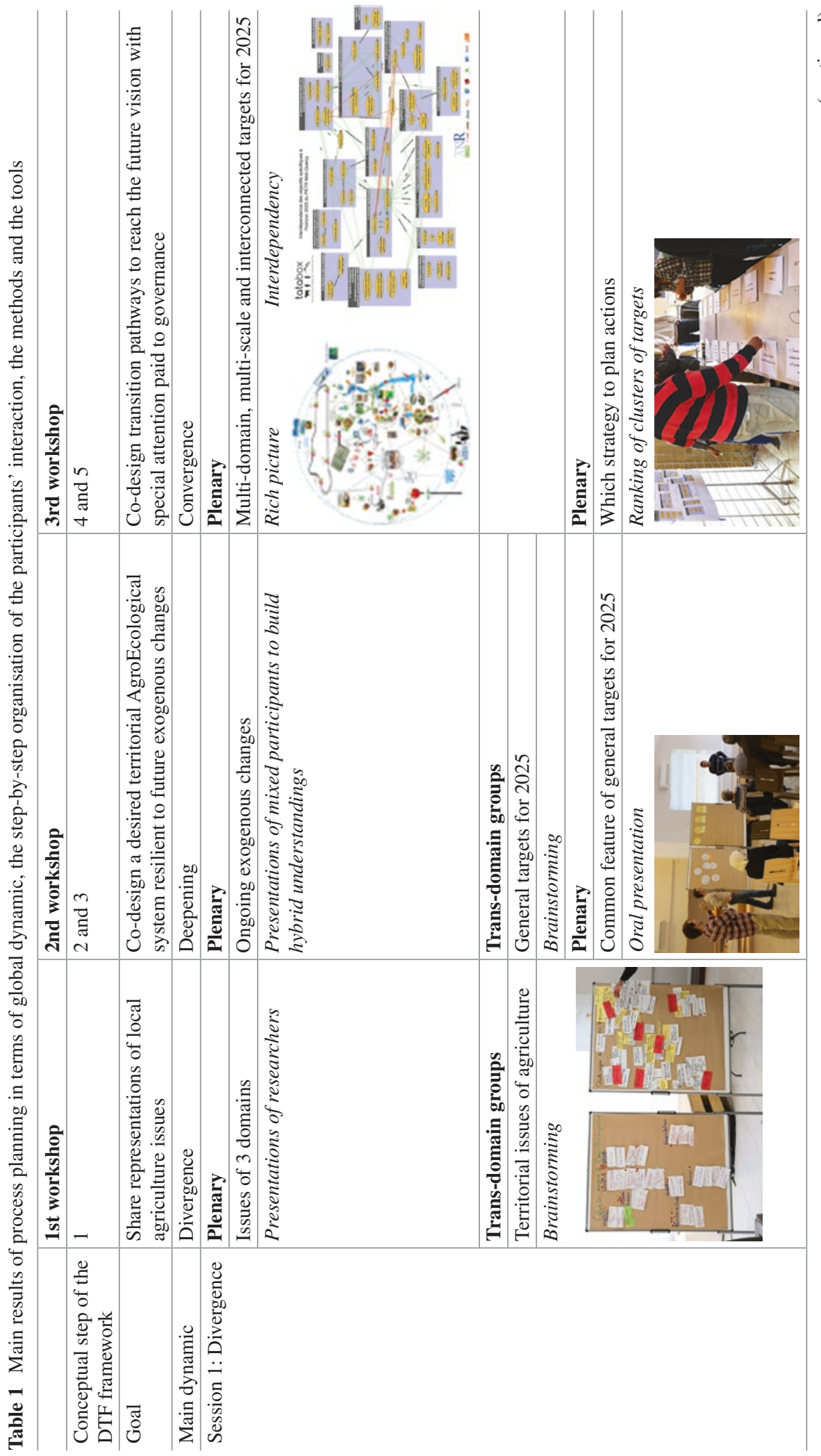




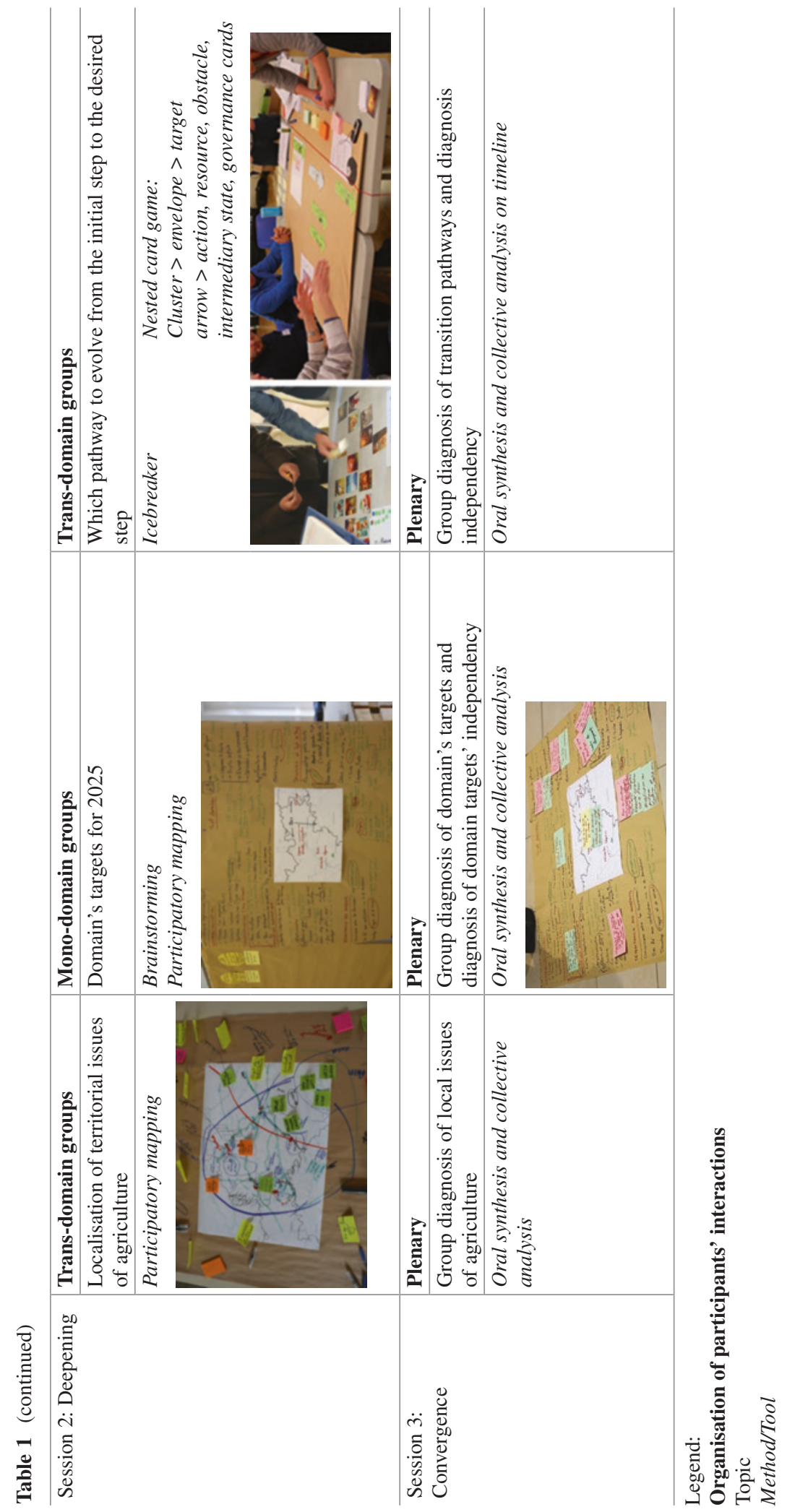


rich picturing. Deepening and convergence were based on concrete representations such as participatory mapping or nested card games reproducing a detailed action plan on a timescale with a playful and interactive format.

\section{Intermediary Tools}

To illustrate original intermediary tools designed for the TATA-BOX process, we focus on a description of Workshop 3, Session 2 (cf. Table 1), in which the objective was to produce a detailed description of the design pathway.

To reach this objective of the workshop session, we developed an original tool (cf. Fig. 7). Each target of the desired vision identified during the second workshop has been symbolised by an arrow (65 targets for Midi-Quercy, 83 for Centre Ouest Aveyron). The target arrow's colour corresponded to a domain group as identified during Workshop 2. The arrow's centre indicated what the target was about and the desired orientation feature (increase $\nearrow$, keep the same $=$, decrease $\searrow$ ). Icons were created for each target to improve its re-appropriation and handling by participants. The icons were all gathered in another intermediary tool: a rich picture providing a global overview of the desired future (cf. Section "Rich picture of a shared vision for 2025"). The ends of the arrows represented the known current state (Workshop 1) and the final desired state (Workshop 2).

Target arrows were clustered into thematic envelopes. The transition pathway was designed one envelope at a time. This work session organisation was intended to facilitate the progressive and incremental analysis of a large numbers of targets.

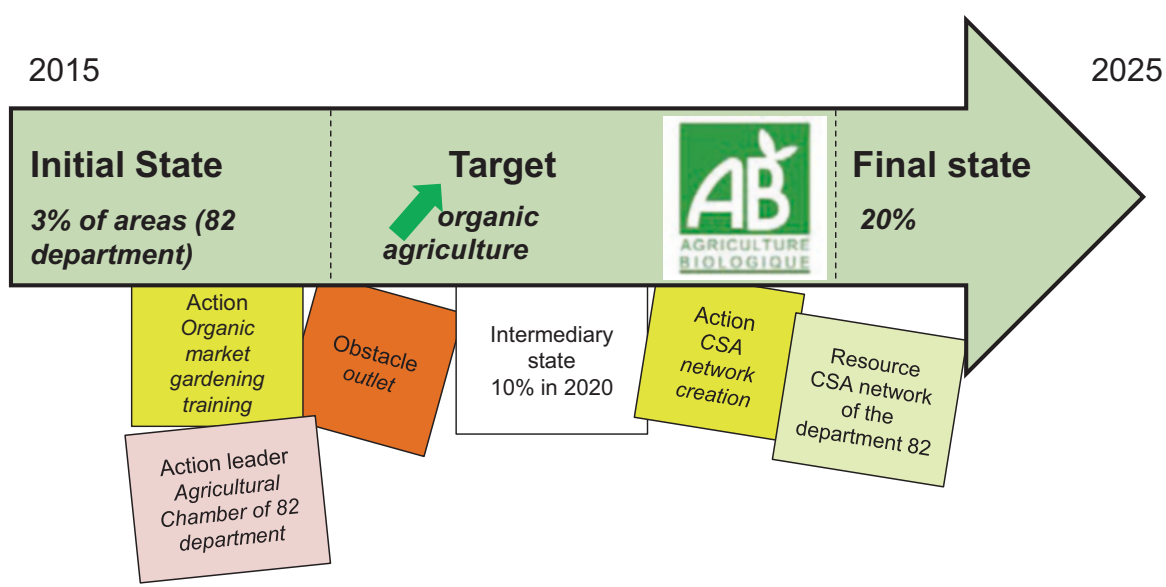

Fig. 7 Card game to examine and detail transition pathways

The transition pathway card game is made up of a set of target-arrows designed from Workshop 2 outcomes: Action, Resource, Obstacle, Action leader and Intermediary states cards used as milestones from the current to the desired state of the target 
The first step of the exercise was designed to help participants to define the adapted temporal sequence for reaching the different targets through the positioning of arrows on a virtual time line, in order to reflect the expected general chronology. They were then asked to detail the strategy to reach each target, using cards of different colours to identify respectively: actions (yellow), possible obstacles (orange) to overcome, resources (green) for action, action leaders (pink), and key intermediary states to monitor between the current state and the expected future one. Participants were free to deal with the cards in any order. Information extracted from Workshop 2 outputs provided participants with predefined cards (e.g. actions, obstacles, resources) but they were free to create new cards and to rule out the predefined ones. Identified links between target arrows were materialised by a sticker, referring to the related target code number.

This exercise was performed in a trans-domain group session. A final plenary session presented the group's main outputs and initiated reflection on a global timeline (cf. Table 1). The aim was to sketch a general action plan by arranging the removable arrows and cards on a vertical support. This reflection continued by reporting a global overview of the arrows and cards on a Gantt diagram (cf. section "Action plan"). The overall chronological organisation was based on the resulting general action plan and could be adjusted on the basis of the dates indicated on intermediary state cards.

\section{Operational Results}

The application of the TATA-BOX participatory design process led to numerous varied operational outcomes. This method supported the formalisation of a shared diagnosis, original transversal targets, the action plan, and associated governance. To illustrate these outcomes, we present three operational outputs: the rich picture, the action plan, and the main effects in the field.

\section{Rich Picture of a Shared Vision for 2025}

During the second workshop the participants detailed a shared vision of local agriculture on the 2025 time line. This vision was described through the identification of 65-83 targets respectively for Midi-Quercy and Centre-Ouest Aveyron.

To synthesise and make more easily accessible the richness of these two visions, scientists developed two corresponding integrative "rich picturing" representations (cf. Fig. 8).

Apart from individual targets, the Rich Picture served to highlight targets' links. Some of them were situated during a participatory mapping exercise (cf. Table 1). The three key organisational levels accounted for by participants were symbolised by nested circles: Farm, Territory, Country (France). These levels were crossed by inflows and outflows. An initial orientation of the picture was set, and all the targets 


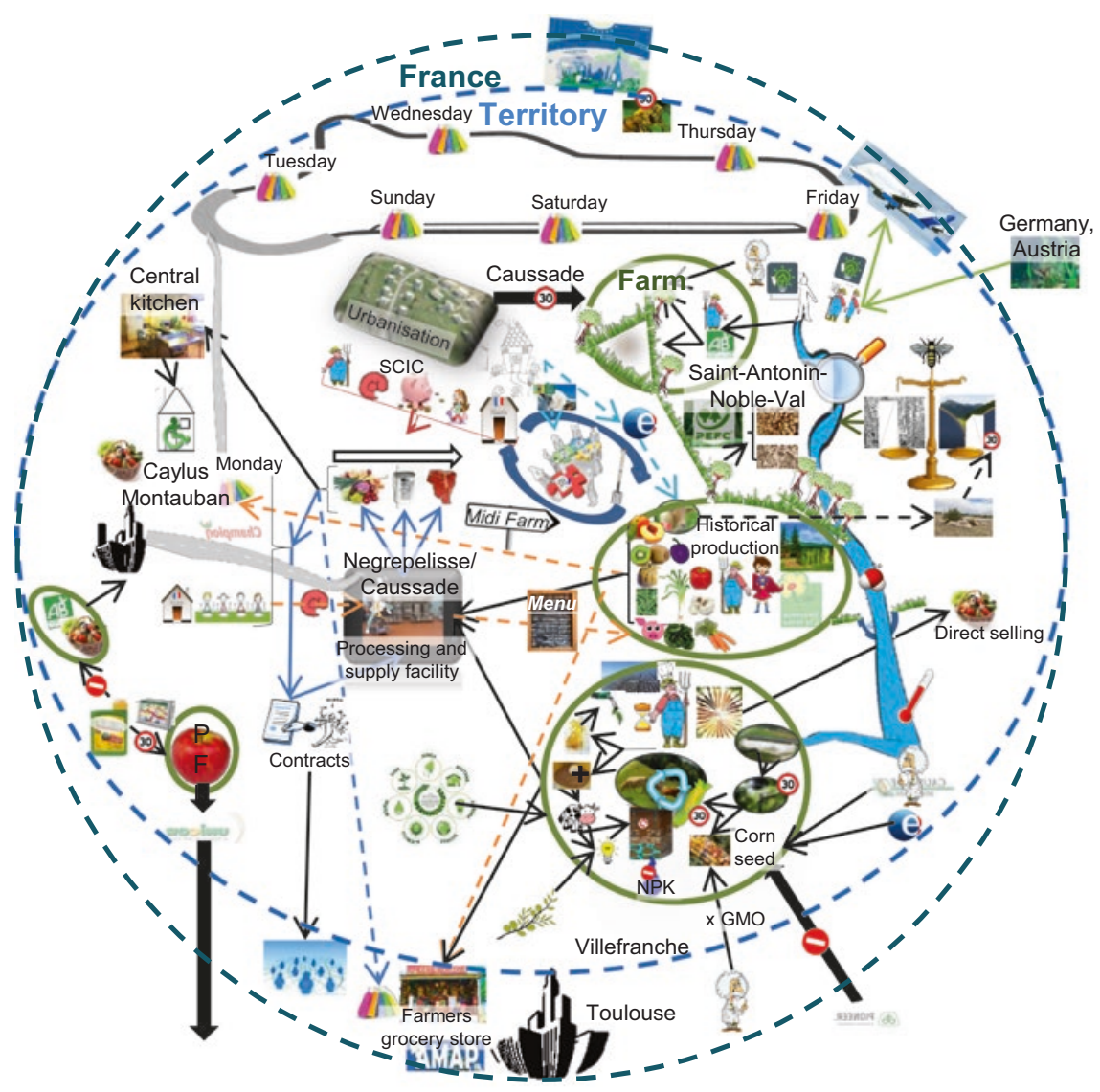

Fig. 8 Rich picture presenting the 65 targets for agriculture in 2025 in Midi-Quercy PETR

were situated on the picture to reflect their geographical embeddedness and to draft a basis for landscape planning.

This output from Workshop 2 was used during the introduction to Workshop 3, to summarise and detail all the outputs of the previous workshop. The facilitator zoomed in on each zone of the rich picture and commented on it. Participants could then react to this representation to highlight agreements, disagreements or obsolete targets since last workshop. The rich picture was displayed throughout the third workshop to keep an overview on the targeted future while detailing transition pathways.

\section{Action Plan}

In the third workshop the participants detailed transition pathways from initial states (Workshop 1) to final desired state (targets identified in Workshop 2) (cf. Fig. 9). 


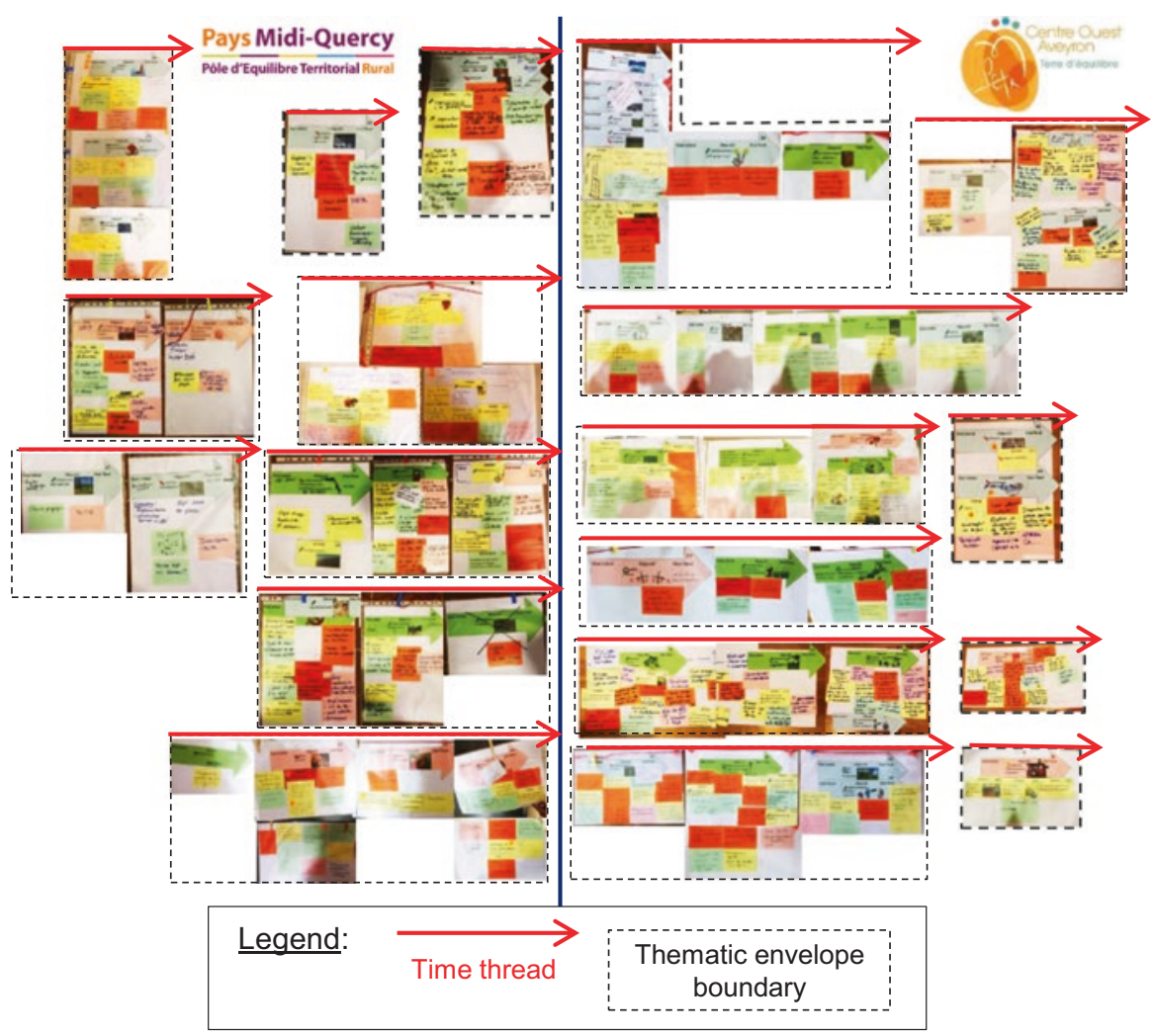

Fig. 9 Overview of third workshop outputs for Midi-Quercy (left side) and Centre-Ouest Aveyron (right side)

Each arrow represents targets; they are sequenced chronologically. Action plans were drawn for each target arrow with action, action leader, resource, obstacle and intermediary states cards

After the workshop, scientists translated this action plan into a Gantt diagram at short, medium and long term (Fig. 10).

This systemic normative foresight led to a large provisional action plan that included numerous axes and action scales, in accordance with the three domains considered: farming system (green arrows in Fig. 9), supply chain (pink), natural resources (blue). 83 and 100 actions were planned respectively for Midi-Quercy and Centre Ouest Aveyron counties, and distributed over the 2016-2025 period (cf. Fig. 10). 28 action leaders in charge of the governance of corresponding actions were identified in each territory respectively (in pink).

Workshop participants had free access to the action plan and could add comments or modify it online.

A second table summarised each organisation's involvement for each target, so that they would have access to information on the actions relevant to them. 


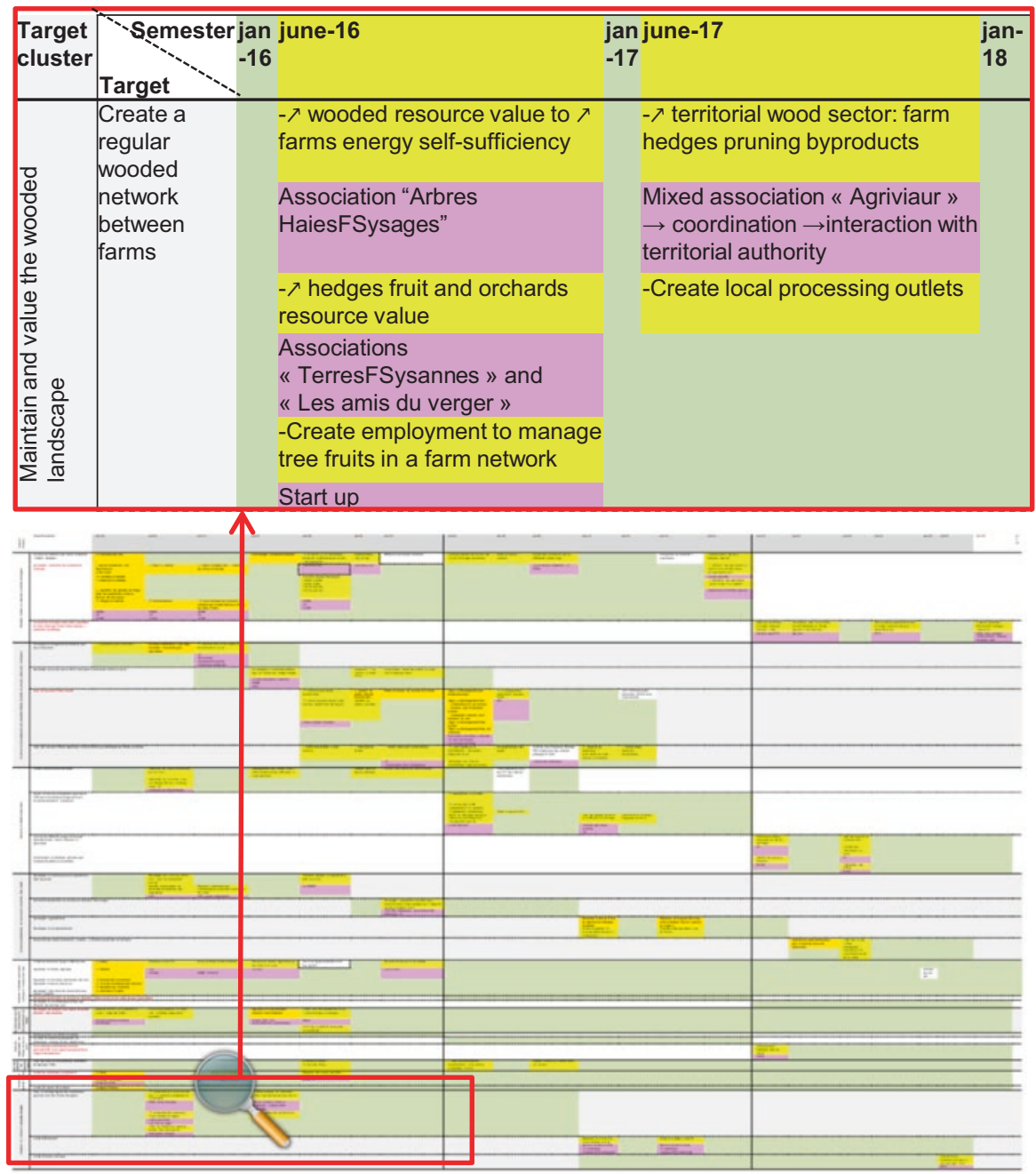

Fig. 10 Detailed action plan of Centre Ouest Aveyron, for each detailed target within each cluster target, transition pathway between the initial and the final state: actions, governance, intermediary states

\section{Implementation of the Methodological Results and Workshop Outputs}

Local partners used some of the outcomes and representation tools for local policies and their own animation activities, respectively. For example, the three illustrated workshop reports were used as a basis for the Territorial Coherence Scheme. Development agents also used representation tools such as the animated "rich picture" during meetings on the development of a Territorial Food Plan. 
Local partners quickly started to implement some of the actions identified in Workshop 3. For example, Midi-Quercy PETR engaged a process to create a Social Cooperatives of General Interest dedicated to renewable energy, as identified in the action plan.

To improve action plan implementation and go further in the process of the transition management, local partners asked scientists to organise a fourth workshop. After discussions between scientists and local partners, the main assigned goals of the workshop were: (i) to share how PETR have used workshop outcomes to this day; (ii) to develop a shared analysis of the particularities and complementarities of action plans in each partner's territory; (iii) to identify possible synergies between both action plans in order to foster their implementation; and (iv) to plan collaborative actions involving both territories. This fourth workshop was entirely designed with local partners.

During this workshop, initially not planned by scientists, local partners built a common strategy, including 11 potential cooperation axes. Like the outcomes of the previous workshops, the corresponding action plan and the required resources were formalised in a formal report.

Finally, the TATA-BOX process also had some indirect impacts concerning participants' networks and their position on AET (cf. chapter "The Key Role of Actors in the Agroecological Transition of Farmers: A Case-Study in the Tarn-Aveyron Basin"). These results and the assessment of the extent to which participants' expectations had been met are detailed in chapter "Evaluation of the Operationalisation of the TATA-BOX Process".

\section{Discussion}

\section{Can the Initial DTF Framework Be Translated into Operational Tools to Design Transition Toward a Territorial AgroEcological System?}

The original conceptual and methodological framework considered three domains and five steps to address major AET issues (cf. Figs. 1 and 2) by means of redesign. This section discusses the extent to which the original frameworks were finally operationalised.

\section{Did the Operational Process Reflect Major Agroecological Transition Issues?}

For a systemic and resilient AET, the DTF framework considered key transition dimensions to be tackled in AET design. Many of the topics included in the initial expectation (Bergez et al. 2013; Duru et al. 2015) were addressed in both territories throughout the participatory process: 
- Biodiversity was considered in the natural resource domain in both territories, with a focus mainly on conservation issues. The topic was also mentioned when dealing with issues of the farming system and supply chain domains, through targets concerning diversification of cropping, farming and landscape systems and food chains. For example, it was addressed in the final action plan through targets like "Develop agrobiodiversity", "Insert pasture within forage systems", or "Develop new chains for former outputs". The initial definition of agroecology was however deliberately broad and open, in order not to influence participants' transition design. Therefore, the process was not long enough to get into further details of expected role and effects of diversity, redundancy, connectivity and feed-backs (Biggs et al. 2012). Even if the process did not focus on specifications of interrelationships between climate change, water resources, crop and animal production, these interdependencies were explicitly tackled by the participants.

- Energy transition was considered as the third priority in the action plans of both territories. It was mainly addressed through the objective of farm production diversification, with a special focus on wooded resources for Centre Ouest Aveyron, and a larger range of renewable energy sources for Midi-Quercy: hydraulic, photovoltaic, solar panels, methanation, and seaweeds.

- The reduction of anthropogenic inputs was an explicit target in the action plan of Centre Ouest Aveyron. In this territory, the issue was closely related to the promotion of farming system autonomy by increased agrobiodiversity ("Insert pasture within forage systems"). For Midi-Quercy, it was not central but was mentioned in relation to water quality.

- Anthropogenic inputs reduction and biodiversity topics were closely related to the development of ecosystem services. However, the term "ecosystemic services" was not used during the first three workshops. The fourth workshop took place with local partners. During this inter-territorial interaction, the participants finally used this term and tackled eco-economy to insert TATA-BOX outcomes within local public policies.

\section{Did the Operational Process Reflect the Three Targeted Domains?}

The design process explicitly concerned the farm system, supply chain and natural resources domains and their interactions. In a divergence-deepening-convergence process, domains were formalised and treated separately in Workshop 2 (vision) while they were treated transversally in Workshop 3 (transition pathways) (cf. Section "Methods to foster participants' interaction".). The rate of actions transversal to at least two domains diverged from one territory to the other. In Midi-Quercy, $3 / 11$ action plan axes mixed two or three domains while this rate reached 7/11 axes in Centre-Ouest Aveyron. Domains were materialised in order to take into account and develop a co-evolution of technological, technical, social, economic and institutional dimensions, with a view to improving the overall sustainability. We actually developed a trans-domain action plan that might favour a multi-dimensional coevolution in the resulting transition. 


\section{Did We Develop a Functional Process, Methods and Tools for Redesign?}

The TATA-BOX research process enabled scientists to develop operational tools to equip local partners for territorial planning. The choice, sequencing and application of methods and tools in the participatory process were helpful to broaden participants' innovation ability while leading them towards a concrete action plan. Compared to other participatory foresights, TATA-BOX had the particularity of enabling participants to design precisely the transition pathways to be implemented at local level. The methods and tools were designed to make this heavier operationalisation step more interactive and didactic, through a one-day workshop.

The tested participatory design process, methods and tools enabled local stakeholders to design a shared provisional action plan with potential action leaders. Additional multi-medium reports on methods were provided to local stakeholders to hand over the methodology: raw intermediary tools; a comic book on key issues of a participatory approach for AET (Audouin et al. 2018b), and a methodology guide for the whole process (Audouin et al. 2018a).

The Scientist-Territories interface could nevertheless have been intensified during the process by additional collaborative tools to develop stakeholder-stakeholder and stakeholder-researcher interaction to foster interaction and methodological adaptations (cf. chapter "Information and Communication Technology (ICT) and the Agroecological Transition").

\section{What Is the Role of Adaptive Multilevel Governance?}

Multi-form and multi-level governance features may strengthen the adaptive capacities of governance systems by increasing the ability to change and ultimately the resilience of the managed social-ecological system (Biggs et al. 2012). Polycentric governance should be multi-level in order to be resilient to change and to address the complexity of the social-ecological system (Pahl-Wostl 2009; Pahl-Wostl et al. 2010; Folke et al. 2011).

The two action plans designed by local stakeholders were based on such multiform and multi-level governance, with a total of 28 identified organisations in charge of actions in each territory, acting at different levels, in different domains, and dealing with different issues. In other words, these action plans were based on a poly-centric governance based on different arenas dealing with specific issues at the organisational level at which they emerge. Importantly, local authorities and territorial policies were identified to drive some of the individual actions constituting the global action plan.

Some of the planned actions had to be led by various complementary organisations (33\% in Midi-Quercy; 79\% in Centre-Ouest Aveyron). The interaction modalities between these existing organisations and the corresponding governance system were however not completely informed. Other actions required the creation of new structures (31\% in Midi-Quercy; 8\% in Centre Ouest Aveyron). For example, Centre-Ouest Aveyron identified the need for a new local business owner to manage 
the wooded resource and derive value from fruit and hedge pruning in a farm network. Midi-Quercy identified a need for two new Social Cooperatives of General Interest (SCIC) dedicated to a local supply-chain platform and local renewable energies coordination. SCIC structures have the specificity to potentially gather farmers as well as elected representative in a same governance structure. Midi-Quercy also considered the creation of an Economic and Environmental Interest Group (GIEE) devoted to organic matter exchanges between livestock farmers and cereal farmers, as well as a bulk purchase group for water resources equipment. An interterritorial governance organisation was proposed for water resource management.

\section{How to Increase the Impact of the Participatory Process on Local Territories?}

While DTF (2015) argued for the necessity to iterate the design process, the TATABOX project allowed for only one iteration. The cycle implemented during the TATA-BOX project resulted in two final action plans collectively designed by workshop participants. These action plans are at once multi-domain, multi-stakeholder, and multi-level, and involve many different biophysical and socio-economics items and variables (cf. Section "Did the operational process reflect major agroecological transition issues?").

Additional steps would be relevant to deepen action plan outcomes in order to facilitate their implementation. These workshops could: (i) deepen action operationalisation modalities and address remaining uncertainties, and (ii) address governance issues and deepen management strategies for the aforesaid actions.

While computer-based models are often used in the design process of cropping and farming systems (Bergez et al. 2010; Martin et al. 2012; Duru et al. 2015), the use of such models to deal with the full complexity of the design process presented here seems difficult or even impossible. It was not possible to use this type of tool to analyse in greater depth the impacts and trade-offs of the various transition pathways. Additional workshops focusing on specific topics and considering a reduced number of variables would however make the use of computer models possible. For example, as DTF (2015) have highlighted, agent-based models could help to address place-based interactions between human decisions and ecological processes at landscape level. GIS- and indicators-based approaches could also be used to deal with issues at field, farm or landscape level.

Adaptive management considers the iterative design-action-monitoring process in order to improve practices and policies by identifying and taking advantage of learning from implementation (Pahl-Wostl 2009). As workshop participants were not individually empowered to deal with such management strategies, additional workshops would have been necessary to enable participants to refine action plan management strategies step-by-step and to refine diversified intertwined multilevel governance systems. 
More generally, while these additional workshops might empower the stakeholders in action plan implementation, new relevant stakeholders, especially operators of technologies and techniques, would need to be included throughout the additional process steps, according to the particularities of the workshop theme. New intermediary tools needed to be developed to support the stakeholder's activities during these meetings. However, this raised the question of the role of TATA-BOX's scientists in this process of transition implementation, as the researchers' withdrawal from the local process was planned at the end of the third workshop. The translation of action plans into local projects required the stakeholders to sort actions into different projects, to find project resources, and to set a final project agenda. These projects were a cornerstone between planning and implementation that actually impacted the other workshop participants. Because the PETR ${ }^{2}$ were identified as key stakeholders of action plan governance in both territories, this cornerstone now entirely relies on PETR ${ }^{2}$ coordination and political will (cf. chapter "Evaluation of the Operationalisation of the TATA-BOX Process"). From the beginning of the project we assumed that the PETR ${ }^{2}$, as local partners, would take in charge the coordination of further steps, and we made sure to equip them with ready-to-use results and the appropriate tools to do so (cf. sections "Scientific design of the participatory methodology" and "Did we develop a functional process, methods and tools for redesign?"). We also complied with their request to organise an additional fourth workshop for exchange and coordination between the two PETR on the possible further steps they would take. Compared to the breadth of the action plan, the actual steps taken by the PETR subsequent to the workshops have however been minimal. Our final question concerns scientists' role in PETR $^{2}$ empowerment in further steps. Should the scientists of the TATA-BOX project wait for the PETR $^{2}$ to switch on their own from transition arena workshop participants to transition governance status? Or which additional tools could scientists provide them with to make this status transition? Did the researchers' withdrawal from the transition process take place at the right time or should they have carried on their maieutic support within the transition process?

\section{What Does It Mean for Scientists?}

\section{Developing Trans-Disciplinary Research}

TATA-BOX was a research project involving a group of 42 researchers. It was clearly defined that the scientific issues were methodological and consisted in testing methods with actors for support and not prescription purposes. As we have seen, the initial DTF framework considered three main areas: "farming systems", "natural resources"and "supply chain". None of the researchers were specialists in all domains. Through our training and individual research, we could provide insights and knowledge on certain themes, but not on the coherence of the whole. The facilitation methods implemented allowed us to introduce new methods and to add the 
researchers' scientific knowledge to the empirical knowledge of the local actors. The group of researchers wanted the participants to articulate local expertise and knowledge, consistent with a post-normal research approach (Funtowicz and Ravetz 1993). Multi-disciplinarity allowed it to delve deeper into emerging themes while mobilising the global systemic vision of the local actors of the territory during the integration of the different domains. Moreover, because of the initial transparency of the researchers' position in the process, and the purpose of the device itself, their disengagement at the end of the project was explicitly discussed and planned with the partners in the field.

\section{“Cheating" to Propose the Project}

The structure of research projects can be a real obstacle to their development in territorial co-design. In the "idealised" scheme of the National Research Agency, knowledge of a partnership and the issue to be addressed is one of the keys to successful acceptance of the project. However, for research topics like the one proposed by TATA-BOX, the partnership is built along the way, and the question - often vague (ill-structured) at the beginning of the project - is clarified over time. The same goes for the choice of methods. Here, we admit, we "cheated". We proposed a project in traditional task groups, but from the first meetings of the scientists, we modified the overall structure of the project to allow for an adaptive research schedule. Moreover, we tried an adhocratic type of governance (which allowed us to be more consistent with our wish to favour the emergence of a poly-centric adaptive governance of the territories, cf. chapter "Towards a Reflective Approach to Research Project Management"). This was difficult to maintain over time because a researcher's reasons for participating in a project vary (financial interest, management, interest in the method/thematic, network, etc.) and their availability evolves. As a result, their degree of involvement in the project varies as well. We therefore maintained a fixed trinomial of facilitators rather than a circle of facilitators in constant rotation.

\section{Conclusion}

The TATA-BOX project aimed at developing a participatory toolbox to support local stakeholders in the design of an AET at local level. Considering the objectives of agroecology, that is, the development of diversified agricultural systems providing ecosystem services that drastically reduce the use of industrial inputs, a "redesign" transition strategy was targeted rather than so-called "efficiency" or "substitution" strategies. The purpose of the TATA-BOX project was the operationalisation of the conceptual and methodological frameworks proposed by DTF (2015) for designing an AET. These authors claim that the design of an AET requires reconfiguration of the stakeholders and resource systems emerging from the interaction between farming systems, supply-chains and natural resources management 
strategies. Their methodological framework was designed to support local stakeholders in steering the AET of these three interdependent domains. The targeted TAES should be resilient to exogenous drivers owing to a multi-domain perspective and adaptive governance.

From theory to practice, the TATA-BOX project effectively succeeded in creating an operational and replicable participatory methodology based on the DTF frameworks. It was tested in two adjacent territories of south-western France (MidiQuercy and Centre-Ouest Aveyron counties). The methodology is structured around three main participatory workshops enabling stakeholders to perform exploratory, normative and backcasting prospective analyses over a medium-term, 10-year period. Each step was organised through a divergence-deepening-convergence process, in which stakeholders' interactions were structured in mono-domain and trans-domain groups. The process was based on the use of different intermediary tools favouring innovative, realistic propositions, individual appropriation and exchange of information. Special attention was paid to equitable shared outputs by means of speaking-time sharing and multi-modal communication.

The workshops resulted in turnkey outputs for local stakeholders, i.e. shared agricultural diagnosis for 2015, a vision for 2025's agroecological territorial system, and a projected action plan for transition from the initial to the final desired agriculture organisation. The projected action plans included about 100 actions each, suited to the territory considered, and the associated action leaders, i.e. the governance structure.

The workshops' outputs actually reflected local particularities through various strategies and trajectories, depending on the territory considered. The analysis of current and future agricultural organisation, based on the characteristics of and interactions between three DTF domains - farming system, supply chain, and natural resources management - have proved to be helpful for stakeholders.

Stakeholders identified other indirect results such as widening networks or crossed learning.

The TATA-BOX project organised only one iteration of the design cycle: diagnostic, normative forecasting, and backcasting. As DTF (2015) expected, other iterations or additional steps would enable stakeholders to improve step-by-step transition design and adaptive governance.

Although this was a process of normative forecasting, it was large enough to adopt a free and holistic approach to transition trajectories. The problematic was too large to apply computer-based models to obtain more details on potential impacts and performances of the desired agriculture vision. More in-depth analysis on certain actions may now be investigated using such modelling tools.

The TATA-BOX process acted as a maieutic support within the transition process. Scientists supported the co-design of an action plan, but left its implementation, monitoring and management (including an iterative and continuous design process) up to the stakeholders. Partners and local stakeholders' commitment in the transition implementation and management could be encouraged by means of appropriate procedures and tools for operational adaptive governance and management of implemented transitions. The story of developing a methodology to support transition is to be continued! 


\section{References}

Audouin E, Bergez JE, Choisis JP et al (2018a) Petit guide de l'accompagnement à la conception collective d' une transition agroécologique à l' échelle du territoire. Toulouse

Audouin E, Bergez JE, Therond O (2018b) TATA-BOX, Neuf métaphores des concepts clefs des démarches participatives pour la transition agroécologique

Bergez JE, Colbach N, Crespo O et al (2010) Designing crop management systems by simulation. Eur J Agron 32:3-9

Bergez JE, Sarthou JP, Soulignac V et al (2013) Programme Agrobiosphère édition 2013, Projet TATA-BOX, Document Scientifique. Toulouse

Biggs R, Schlüter M, Biggs D et al (2012) Toward principles for enhancing the resilience of ecosystem services. Annu Rev Environ Resour 37:421-444

Bryson J, Quick KS, Slotterback CS, Crosby BC (2012) Designing public participation process. Public Adm Rev 73:23-34. https://doi.org/10.1111/j.1540-6210.2012.02678.x

Cash DW, Clark WC, Alcock F et al (2003) Knowledge systems for sustainable development. Proc Natl Acad Sci U S A 100:8086-8091

Checkland P, Poulter J (2006) Learning for action: a short definitive account of soft systems methodology and its use, for practitioners, Teachers and Students. Wiley, Chichester

Duru M, Therond O, Fares M (2015) Designing agroecological transitions; a review. Agron Sustain Dev 35:1237-1257. https://doi.org/10.1007/s13593-015-0318-x

Folke C, Jansson Å, Rockström J et al (2011) Reconnecting to the biosphere. Ambio 40:719-738. https://doi.org/10.1016/j.gloenvcha.2006.04.002

Foxon T, Reed M, Stringer L (2009) Governing long-term social-ecological change: what can the adaptive management and transition management approaches learn from each other? Environ Policy Gov 2:3-20. https://doi.org/10.1002/eet.496

Funtowicz S, Ravetz JR (1993) Science for the post-normal age. Futures 31:735-755

Grimble R, Wellard K (1997) Stakeholder methodologies in natural resource management: a review of principles, contexts, experiences and opportunities. Agric Syst 55:173-193

Jordan S, Kapoor D (2016) Re-politicizing participatory action research: unmasking neoliberalism and the illusions of participation. Educ Action Res 24:134-149. https://doi.org/10.1080/0965 0792.2015 .1105145

Lane DC (1998) The greater whole : towards a synthesis of system dynamics and soft systems methodology. Eur J 2217:214-235

Martin G, Martin-Clouaire R, Duru M (2012) Farming system design to feed the changing world. A review. Agron Sustain Dev 33:131-149. https://doi.org/10.1007/s13593-011-0075-4

Moraine M, Mélac P, Ryschawy J et al (2017) A participatory method for the design and integrated assessment of crop-livestock systems in farmers' groups. Ecol Indic 72:340-351

Pahl-Wostl C (2009) A conceptual framework for analysing adaptive capacity and multi-level learning processes in resource governance regimes. Glob Environ Chang 18:354-365. https:// doi.org/10.1016/j.gloenvcha.2009.06.001

Pahl-Wostl C, Holtz G, Kastens B, Knieper C (2010) Analyzing complex water governance regimes: the management and transition framework. Environ Sci Pol 13:571-581. https://doi. org/10.1016/j.envsci.2010.08.006

Pinto-Correia T, Gustavsson R, Pirnat J (2006) Bridging the gap between centrally defined policies and local decisions: towards more sensitive and creative rural landscape management. Landsc Ecol 21:333-346

Pretty JN (1995) Participatory learning for sustainable agriculture. World Dev 23:1247-1263

Shucksmith M (2010) Disintegrated rural development? Neoendogenous rural development, planning and place-shaping in diffused power contexts. Sociol Rural 50:1-14

Therond O, Paillard D, Bergez JE, et al (2010) From farm, landscape and territory analysis to scenario exercise: an educational programme on participatory integrated analysis. In: IFSA symposium, 4-7 july 2010, Vienna, Austria. pp 2206-2216 
Vergne A (2013) Qualité de la participation. In: CASILLO I. avec BARBIER R., BLONDIAUX L., CHATEAURAYNAUD F., FOURNIAU J-M., LEFEBVRE R. NC et SD (dir. . (ed) Dictionnaire critique et interdisciplinaire de la participation. GIS Démocratie et Participation, Paris

Vinck D (2009) De l'objet intermédiaire à l'objet-frontière, Vers la prise en compte du travail d'équipement. Rev d'anthropologie des connaissances 3:51-72

Vinck D (2011) Taking intermediary objects and equipping work into account in the study of engineering practices. Eng Stud 3:25-44. https://doi.org/10.1080/19378629.2010.547989

Open Access This chapter is licensed under the terms of the Creative Commons Attribution 4.0 International License (http://creativecommons.org/licenses/by/4.0/), which permits use, sharing, adaptation, distribution and reproduction in any medium or format, as long as you give appropriate credit to the original author(s) and the source, provide a link to the Creative Commons licence and indicate if changes were made.

The images or other third party material in this chapter are included in the chapter's Creative Commons licence, unless indicated otherwise in a credit line to the material. If material is not included in the chapter's Creative Commons licence and your intended use is not permitted by statutory regulation or exceeds the permitted use, you will need to obtain permission directly from the copyright holder.

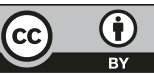

